\title{
A enfermagem no cuidado paliativo domiciliar de idosos apoiada por avaliações multidimensionais: revisão integrativa
}

\author{
Nursing actions in home palliative care for the elderly supported by multidimensional assessments: \\ integrative review
}

Actuaciones de enfermería en cuidados paliativos domiciliarios del anciano apoyados en valoraciones multidimensionales: revisión integradora

Thiago de Medeiros Souza ORCID: https://orcid.org./0000-0002-8222-917X Universidade Federal de Juiz de Fora, Brasil E-mail: thiagomedrj@gmail.com

Luana Alves e Costa Soares ORCID: https://orcid.org/0000-0002-5369-3811 Universidade Federal de Juiz de Fora, Brasil

E-mail: luanaalves.costasoares@gmail.com

Suellen de Souza Barbosa ORCID: https://orcid.org/0000-0002-9600-9784 Universidade Federal de Juiz de Fora, Brasil E-mail: suellensouza721@gmail.com

Sandra Trindade Toledo

ORCID: https://orcid.org/0000-0001-9486-3939 Universidade Federal de Juiz de Fora, Brasil E-mail: sandra.t.toledo@gmail.com

Mariana Santos Rezende ORCID: https://orcid.org/0000-0003-2357-8908 Universidade Federal de Juiz de Fora, Brasil E-mail: marianarezende010@gmail.com

Edna Aparecida Barbosa de Castro ORCID: https://orcid.org/0000-0001-9555-1996 Universidade Federal de Juiz de Fora, Brasil E-mail: ednabdecastro@aol.com

\begin{abstract}
Resumo
Objetivo: Analisar as ações da enfermagem que são sistematizadas pelo emprego de instrumentos de avaliação multidimensionais durante a assistência de cuidados paliativos ao idoso no domicílio. Método: Revisão integrativa de artigos publicados entre 2011 e 2020, identificados nas bases MEDLINE, LILACS, BVS, BDENF e IBECS, CINAHL em língua portuguesa, inglesa ou espanhola; com resumos e/ou texto disponível para leitura na íntegra e com acesso gratuito, buscando responder à questão de pesquisa "No CP de pessoas idosas, a avaliação do enfermeiro utilizando instrumentos de avaliação adotados em outros contextos assistenciais orientam ações da enfermagem no cuidado paliativo domiciliar?". Resultados: A partir das buscas nas bases de dados foram identificados 242 artigos, dos quais 19 foram incluídos no presente estudo, possibilitando a identificação de instrumentos de avaliação multidimensionais e, focos de atuação da enfermagem. Conclusão: Evidenciou-se a abrangência e a complexidade da atuação do enfermeiro no contexto dos cuidados paliativos domiciliares, envolvendo a gestão das necessidades assistenciais e promoção de ações direcionadas, com caráter multidimensional e holístico.
\end{abstract}

Palavras-chave: Cuidados paliativos; Assistência domiciliar; Saúde do idoso; Avaliação em enfermagem; Processo de enfermagem.

\begin{abstract}
Objective: To analyze the nursing actions that are systematized by the use of multidimensional assessment instruments during palliative care assistance to the elderly at home. Method: Integrative review of articles published between 2011 and 2020, identified in the MEDLINE, LILACS, BVS, BDENF and IBECS, CINAHL databases, in Portuguese, English or Spanish; with abstracts and/or text available for full reading and free access, seeking to answer the research question "In the PC for elderly people, the nurse's assessment using assessment instruments adopted in other care contexts guide nursing actions in palliative home care?". Results: From the searches in the databases, 242 articles were identified, of which 19 were included in the present study, enabling the identification of
\end{abstract}


multidimensional assessment instruments and focuses of nursing action. Conclusion: The scope and complexity of nurses' activities in the context of home palliative care was evidenced, involving the management of care needs and the promotion of targeted actions, with a multidimensional and holistic character.

Keywords: Palliative care; Home nursing; Health of the elderly; Nursing assessment; Nursing process.

\section{Resumen}

Objetivo: Analizar las acciones de enfermería que se sistematizan mediante el uso de instrumentos de evaluación multidimensional durante la asistencia en cuidados paliativos al anciano en el domicilio. Método: Revisión integradora de artículos publicados entre 2011 y 2020, identificados en las bases de datos MEDLINE, LILACS, BVS, BDENF e IBECS, CINAHL, en portugués, inglés o español; con resúmenes y / o texto disponibles para lectura íntegra y de libre acceso, buscando dar respuesta a la pregunta de investigación “¿En el AP de ancianos, la valoración del enfermero mediante instrumentos de valoración adoptados en otros contextos asistenciales orientan las acciones de enfermería en cuidados paliativos domiciliarios?". Resultados: De las búsquedas en las bases de datos se identificaron 242 artículos, de los cuales 19 fueron incluidos en el presente estudio, lo que permitió identificar instrumentos de evaluación multidimensional y focos de acción de enfermería. Conclusión: Se evidenció el alcance y la complejidad de las actividades del enfermero en el contexto de los cuidados paliativos domiciliarios, involucrando la gestión de las necesidades de cuidado y la promoción de acciones focalizadas, con un carácter multidimensional y holístico.

Palabras clave: Cuidados paliativo; Atención domiciliaria de salud; Salud del anciano; Evaluación en enfermería; Proceso de enfermería.

\section{Introdução}

Os cuidados paliativos (CP) são definidos pela Organização Mundial da Saúde como um meio de trazer conforto e melhorar a qualidade de vida de pessoas com doenças ameaçadoras da vida, pela prevenção e alívio do sofrimento, promoção da dignidade, cuidado integral e sistematizado, garantindo o controle eficiente de sintomas e a compreensão do sofrimento biopsicossocial e espiritual de pacientes e de seus familiares (World Health Organization [WHO], 2018).

O crescente envelhecimento populacional, associado à prevalência de doenças crônicas não transmissíveis e degenerativas em um cenário de pobreza, pouca organização política e dificuldade de acesso aos serviços de saúde, fazem com que, aproximadamente, $40 \%$ dos pacientes que necessitem de CP no mundo, pertençam ao grupo das pessoas com 70 anos ou mais de idade. Esta constatação, põe em evidência a demanda por estruturas e organizações que acompanhem indivíduos com doenças progressivas e incuráveis na busca por dignidade de atendimento às suas questões de saúde, baseadas em diferentes modalidades assistenciais (WHO, 2014).

Não obstante, no Brasil, observa-se uma complexa mudança no comportamento de saúde e adoecimento da população devido a um conjunto de transformações sociais, econômicas e principalmente demográficas, destacando-se a população idosa (Perreira, Jesus, \& Martins, 2020) Há uma epidemia de doenças crônicas não degenerativas que expõe a população de baixa renda a diversos fatores de risco e, acrescenta-se a menor oportunidade em acessar serviços de saúde e práticas de promoção à saúde e prevenção de doenças, culminando em extrema vulnerabilidade (Malta et al., 2017).

Em paralelo, o grau de vulnerabilidade do idoso é comumente representado pelo termo "fragilidade" e, considerando o adoecimento desse grupo, aliado ao processo biológico de envelhecimento, há uma potencialização das condições de fragilidade, incapacidades e perda de autonomia, deixando este grupo vulnerável e, na maioria das vezes, cronicamente dependente do cuidado de outros (Leandro et al., 2019).

A Política Nacional de Saúde da Pessoa Idosa (Brasil, 2006), considera "idoso frágil ou em situação de fragilidade aquele que: vive em instituição de longa permanência para idosos (ILPI); encontra-se acamado; esteve hospitalizado recentemente por qualquer razão; apresente doenças sabidamente causadoras de incapacidade funcional (acidente vascular encefálico, síndromes demenciais e outras doenças neurodegenerativas, etilismo, neoplasia terminal, amputações de membros); encontra-se com pelo menos uma incapacidade funcional básica ou vivencie situações de violência doméstica” (Brasil, 2006; Torres, Campos, Luiza, \& Caldas, 2020). 
Há um desafio no reconhecimento da fragilidade de pessoas idosas, bem como a implementação de intervenções capazes de atender integralmente às suas necessidades e a atenção domiciliar (AD) representa uma alternativa assistencial com potencial para incluir esta demanda. A avaliação multidimensional é uma estratégia que vem sendo difundida na saúde pública, ancorada no conceito de fragilidade multidimensional, compreendendo que as condições de saúde de pessoas idosas, associadas a desfechos adversos, podem ser agrupadas em dois componentes: clínico-funcional e sociofamiliar, o que valoriza todos os componentes da fragilidade em idosos, entendida como a presença de incapacidades e/ou de condições crônicas preditoras de declínio funcional, institucionalização e/ou óbito (Larissa, Coutinho, \& Teixeira, 2021; Nunes De Moraes, 2012).

Munindo-se desta ótica, os profissionais de saúde que desenvolvem CP para idosos em domicílio tendem a incorporar instrumentos de avaliação geriátrica multidimensionais em suas rotinas de cuidado, com a intenção de facilitar o levantamento das necessidades de saúde desse grupo, o sofrimento biopsicossocial e espiritual, bem como o reconhecimento de situações de risco para declínio funcional e perda de conforto/qualidade de vida, diante do acometimento e evolução da doença crônica (Sanvezzo, Montandon, \& Esteves, 2018).

Por razões semelhantes, nos últimos anos, tem-se observado uma maior utilização de instrumentos de avaliação na prática assistencial do enfermeiro potencializando o achado de alterações biológicas, aferição das progressões, retrocessos ou estagnações de um estado de saúde-doença do paciente, de maneira objetiva, acompanhamento de fenômenos subjetivos uniformizando e dando foco nas etapas do planejamento e intervenções de enfermagem (Gardona \& Barbosa, 2018).

No âmbito assistencial, o processo de cuidar da enfermagem envolve o histórico ou obtenção de informações de saúde; diagnóstico de enfermagem; planejamento; implementação e a avaliação (Conselho Federal de Enfermagem [COFEN], 2009). O cuidado da enfermagem deve ser sistematizado, para que se atinja a resolubilidade das necessidades de saúde da população, qualificação e segurança ao ser ofertado (Adamy, Zocche, \& Almeida, 2020). A avaliação como parte do processo de cuidar é relevante na definição do plano de cuidados, e, em se tratando de pessoas idosas no domicílio, este se se estende também à família (Barbosa, Nascimento, Carvalho, Aoyama, \& Lima, 2019; Leandro et al., 2019).

A prática do enfermeiro, quando circunscrita à área do CP domiciliar de idosos requer especializações, formação e atualizações constantes para a aplicação sistemática do processo de enfermagem que produza um plano de cuidados individualizado e satisfatório a cada etapa de progressão da doença, de modo a otimizar a oferta de cuidados e garantir o conforto adequado. Com a execução do processo de enfermagem, favorece-se a identificação correta dos problemas do idoso, com a eleição dos principais diagnósticos de enfermagem e definição das metas de cuidado (Plano de Cuidados), para então atuar com intervenções efetivas (Caveião et al., 2019; Passarelles, Rios, \& Santana, 2019).

Deste modo, esta revisão se justifica pela crescente e dinâmica transferência dos CP para contextos domiciliares, requerendo exploração, identificação e sistematização dos conhecimentos existentes acerca da avaliação que orienta a atuação da enfermagem, vislumbrando-se ampliação e avanços do conhecimento nesta área de intercessão interdisciplinar. Ressalta-se que no cenário brasileiro são poucas as publicações sobre instrumentos validados e adaptados transculturalmente para investigação multidimensional de pessoas idosas em $\mathrm{CP}$ segundo a perspectiva da $\mathrm{AD}$, cabendo a utilização frequente de instrumentos avaliativos internacionais.

A avaliação e coleta de informações é essencial para o levantamento de problemas (diagnósticos) sensíveis à atuação do enfermeiro, em seu processo de trabalho na AD. Portanto, é relevante estudar qual tem sido o foco da enfermagem na equipe, seja como base para futuras pesquisas e seja para orientar o processo de cuidar do enfermeiro neste contexto, como forma de apoio os esforços feitos para a promoção da qualidade de vida e de segurança de pacientes idosos (coletivo ou família) em CP domiciliar.

Esta revisão tem por objetivo levantar na literatura as ações da enfermagem que são sistematizadas pelo emprego de instrumentos de avaliação multidimensional durante a assistência de $\mathrm{CP}$ ao idoso no domicílio. 


\section{Metodologia}

A revisão integrativa é um método sistemático que permite revisar estudos primários existentes de modo abrangente, planejado e imparcial, com análises críticas dos resultados, identificação de vieses e valorização de evidências clínicas na busca de respostas específicas para uma questão de pesquisa (Brasil, 2014; Donato \& Donato, 2019). É uma abordagem metodológica ampla, que permite incluir estudos experimentais e não-experimentais para uma compreensão completa do fenômeno analisado, capaz de fornecer um panorama consistente e compreensível de condições relevantes para a prática da enfermagem (Whittemore \& Knafl, 2005).

Nesta revisão adotou-se as oito etapas propostas por Chitu Okoli (2019). Na primeira etapa identificou-se a questão e objetivo da pesquisa, e na segunda etapa, fez-se o planejamento e o treinamento da equipe de pesquisa, definindo-se o protocolo.

Com isso, formulou-se a questão de pesquisa com o acrônimo PICO (Quadro1): População, Intervenção, Comparação e "Outcomes" [desfecho] que, no contexto das práticas baseadas em evidências, traz rigor metodológico aos modos de investigação (Brasil, 2014). No CP de pessoas idosas (P), a avaliação do enfermeiro (I) utilizando instrumentos de avaliação adotados em outros contextos assistenciais $(\mathrm{C})$ orientam ações da enfermagem no cuidado paliativo domiciliar $(\mathrm{O})$ ?

Quadro 1 - Elementos da questão de pesquisa segundo o acrônimo PICO, Juiz de Fora, Minas Gerais, Brasil, 2021.

\begin{tabular}{|l|l|}
\hline P & Pessoas idosas \\
\hline I & Avaliação de idosos em cuidado paliativo domiciliar. \\
\hline C & Tipos de instrumentos utilizados em outros cenários \\
\hline O & Ações orientadas pela avaliação para o CP domiciliar \\
\hline
\end{tabular}

Fonte: Adaptado de Brasil (2014).

Definiu-se o ano de 2011 como ponto de partida para a revisão, haja vista a implantação no Brasil, da Política Nacional de Atenção Domiciliar, que incluiu a enfermagem para atuação em um dos seus eixos que desde então tem sido a desospitalização para cuidado paliativo domiciliar (Brasil, 2011). A busca ocorreu entre os meses de março e abril de 2020, utilizando descritores disponíveis no DeCS (Descritores em Ciências da Saúde) e MESH (Medical Subject Headings). Os artigos de interesse deveriam ser encontrados mediante a seguinte combinação: na língua inglesa (("palliative care" AND "Health of the elderly" AND "Home nursing" AND "nursing assessment")) e, em espanhol ((“cuidados paliativos" AND "salud del anciano" AND "atención domiciliaria de salud" AND "evaluacuón en enfermeira")).

Para a busca dos artigos, elegeram-se as seguintes bases de dados: MEDLINE (Medical Literature Analysis and Retrieval System Online); LILACS (Literatura Latino-Americana e do Caribe em Ciências da Saúde); BVS (Biblioteca Virtual em Saúde); BDENF (Banco de Datos de Enfermería); IBECS (Índice Bibliográfico Español en Ciencias de la Salud) e; Cochrane Library Collaboration.

$\mathrm{Na}$ terceira etapa foi feita uma seleção prática dos artigos encontrados com os critérios de inclusão: pesquisas com humanos, na faixa etária de 60 anos ou mais; publicadas no período de 2011 a 2020; em periódicos nacionais e internacionais; em língua portuguesa, inglesa ou espanhola; com resumos e/ou texto disponível para leitura na íntegra, independente do método adotado e com acesso gratuito.

Na quarta e quinta etapas buscaram-se e extraíram-se artigos das bases de dados, extraindo-se 242 artigos. Na sexta etapa pela leitura dos títulos e resumos, exclui-se publicações em duplicidade e avaliou-se a qualidade das publicações 
obtendo-se a seleção primária. Em seguida, na sétima etapa chegou-se à seleção secundária. Após o download gratuito, procedeu-se à leitura superficial e observação dos conteúdos, excluindo-se os que não correlacionavam à temática e objetivo do estudo, resultando em 31 artigos. Com a organização destes em um banco de dados próprio investiu-se na leitura em profundidade e análise crítica, vidando observar a relação entre o conteúdo e a questão de pesquisa, excluindo 12 que não a atenderam. A amostra final foi, portanto, de 19 artigos relidos e analisados.

A oitava e última etapa consistiu na síntese do conhecimento, organização, exposição, discussão, reflexão dos principais resultados obtidos e que trouxeram iluminação à questão investigada. Em paralelo, os artigos selecionados na amostragem final, foram classificados quanto ao nível de evidência pelo sistema GRADE (Grading the quality of evidence and the strength os recommendations) que estabelece critérios de classificação e avaliação da qualidade de evidências científicas disponíveis em artigos analisados (Brasil, 2014).

A Figura 1 representou a estratégia de pesquisa relatada, bem como as seleções primárias e secundárias dos artigos.

Figura 1 - Fluxograma do processo de pesquisa da revisão integrativa.

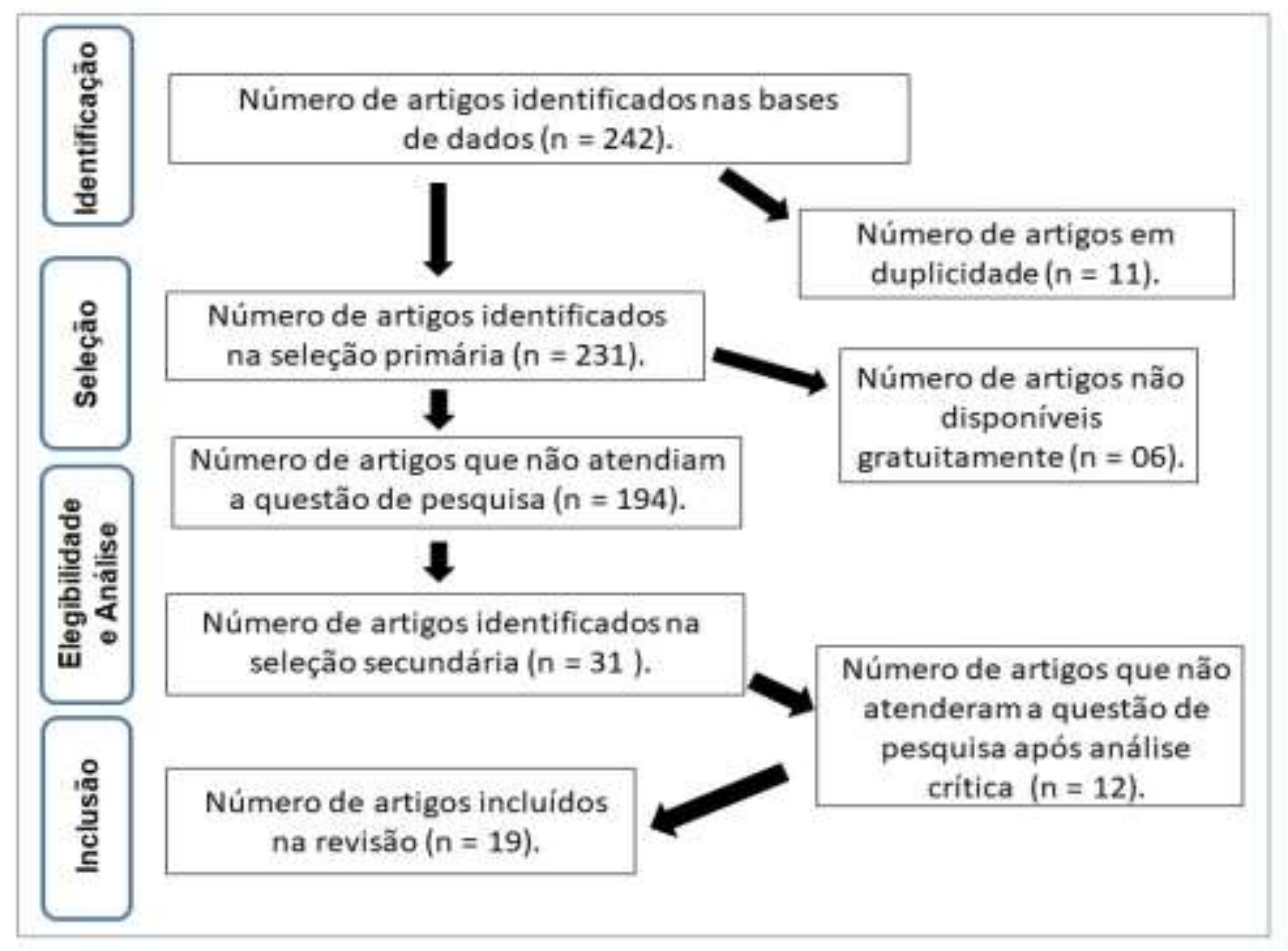

Fonte: Autores, com os dados da pesquisa.

\section{Resultados e discussão}

A matriz criada para registrar as características metodológicas dos estudos incluídos pode ser vista no Quadro 2. 
Quadro 2 - Características dos artigos selecionados para revisão da avaliação de idosos em cuidado paliativo domiciliar pelo enfermeiro.

\begin{tabular}{|c|c|c|c|c|c|}
\hline $\begin{array}{l}\text { Referência / Ano / } \\
\text { Local do estudo / } \\
\text { Periódico / }\end{array}$ & Título do Artigo & Método & Objetivo & $\begin{array}{c}\text { Cenário do } \\
\text { Cuidado }\end{array}$ & $\begin{array}{l}\text { Nível de } \\
\text { Evidência } \\
\text { (GRADE) }\end{array}$ \\
\hline $\begin{array}{c}\text { [01] Burns, M.; } \\
\text { McIlfatrick, S. } \\
\text { /2015/ IRLANDA DO } \\
\text { NORTE/ } \\
\text { International Journal of } \\
\text { Palliative Nursing }\end{array}$ & $\begin{array}{l}\text { Palliative care in } \\
\text { dementia: literature } \\
\text { review of nurses' } \\
\text { knowledge and } \\
\text { 6iloto f towards pain } \\
\text { assessment }\end{array}$ & $\begin{array}{c}\text { Revisão } \\
\text { Sistemática. }\end{array}$ & $\begin{array}{l}\text { Explorar os } \\
\text { conhecimentos e atitudes } \\
\text { dos enfermeiros em } \\
\text { relação à avaliação da dor } \\
\text { em idosos com demência. }\end{array}$ & $\begin{array}{c}\text { Todas as } \\
\text { modalidades } \\
\text { de saúde, a } \\
\text { maioria ILPI }\end{array}$ & Alto \\
\hline $\begin{array}{l}\text { [02] Fristedt, S; Nystedt, } \\
\text { P; Skogar, O. / 2019 / } \\
\text { SUÉCIA / Clinical } \\
\text { Interventions in Aging }\end{array}$ & $\begin{array}{c}\text { Mobile Geriatric Teams } \\
\text { - A Cost-Effective Way } \\
\text { Of Improving Patient } \\
\text { Safety And Reducing } \\
\text { Traditional Healthcare } \\
\text { Utilization Among The } \\
\text { Frail Elderly? A } \\
\text { Randomized Controlled } \\
\text { Trial. }\end{array}$ & $\begin{array}{l}\text { Estudo de } \\
\text { método } \\
\text { misto com } \\
\text { ensaios } \\
\text { clínicos } \\
\text { randomizado } \\
\text { s. }\end{array}$ & $\begin{array}{l}\text { Realizar uma análise de } \\
\text { métodos mistos para } \\
\text { medir a eficácia e a } \\
\text { satisfação do usuário. }\end{array}$ & Domicílio & Alto \\
\hline $\begin{array}{l}\text { [03] Lamppu, P. J. et al. } \\
\text { /2019 / FINLÂNDIA / } \\
\text { European Geriatric } \\
\text { Medicine }\end{array}$ & $\begin{array}{l}\text { Training nursing home } \\
\text { staf to improve 6iloto } \\
\text { f6s' end-of-life care: } \\
\text { design and baseline } \\
\text { fndings from a } \\
\text { randomized controlled } \\
\text { trial }\end{array}$ & $\begin{array}{l}\text { Estudo } \\
\text { randomizado } \\
\text { controlado } \\
\text { por cluster. }\end{array}$ & $\begin{array}{c}\text { Descrever uma } \\
\text { intervenção educacional } \\
\text { sobre cuidados paliativos } \\
\text { realizada com } \\
\text { funcionários de } \\
\text { enfermarias de um lar de } \\
\text { idosos. }\end{array}$ & ILPI & Moderado \\
\hline $\begin{array}{c}\text { [04] Abernethy, A. P. et } \\
\text { al. /2013 / } \\
\text { AUSTRÁLIA / Journal } \\
\text { of pain and symptom } \\
\text { Management }\end{array}$ & $\begin{array}{l}\text { Delivery Strategies to } \\
\text { Optimize Resource } \\
\text { Utilization and } \\
\text { Performance Status for } \\
\text { Patients With Advanced } \\
\text { Life-Limiting Illness: } \\
\text { Results From the } \\
\text { "Palliative Care Trial", }\end{array}$ & $\begin{array}{l}\text { Estudo } \\
\text { randomizado } \\
\text { controlado } \\
\text { por cluster. }\end{array}$ & $\begin{array}{l}\text { Aprimorar os modelos } \\
\text { atuais de provisão de } \\
\text { cuidados paliativos. }\end{array}$ & $\begin{array}{l}\text { Domicílio, } \\
\text { ILPI }\end{array}$ & Moderado \\
\hline $\begin{array}{l}\text { [05] Allen, K. et al. / } \\
2012 \text { / EUA / Popul } \\
\text { Health Manag }\end{array}$ & $\begin{array}{c}\text { The Promoting Effective } \\
\text { Advance Care for Elders } \\
\text { (PEACE) Randomized } \\
\text { Pilot Study: } \\
\text { Theoretical Framework } \\
\text { and Study Design. }\end{array}$ & $\begin{array}{l}\text { Estudo } \\
\text { prospectivor } \\
\text { andomizado. }\end{array}$ & $\begin{array}{c}\text { Descrever as bases } \\
\text { teóricas para } \\
\text { o estudo piloto } \\
\text { randomizado de } \\
\text { Promoção de Cuidados } \\
\text { Avançados Eficazes para } \\
\text { Idosos } \\
\text { (PEACE). }\end{array}$ & Domicílio & Moderado \\
\hline $\begin{array}{c}\text { [06] Harman, L. et al. / } \\
2019 \text { / CANADÁ / BMC } \\
\text { Palliative Care }\end{array}$ & $\begin{array}{c}\text { Potential quality } \\
\text { indicators for seriously } \\
\text { ill } \\
\text { home care 6ilotof: a } \\
\text { cross-sectional analysis } \\
\text { using Resident } \\
\text { Assessment Instrument } \\
\text { for } \\
\text { Home Care (RAI-HC) } \\
\text { data for Ontario. }\end{array}$ & $\begin{array}{l}\text { Revisão de } \\
\text { literatura e } \\
\text { consulta com } \\
\text { especialistas. }\end{array}$ & $\begin{array}{l}\text { Desenvolver um conjunto } \\
\text { de indicadores de } \\
\text { qualidade no cuidado a } \\
\text { idosos em CP domiciliar. }\end{array}$ & Domicílio & Moderado \\
\hline $\begin{array}{l}\text { [07] Hermans, } \text { K. et al. / } \\
\text { 2014/ BÉLGICA/ } \\
\text { JAMDA }\end{array}$ & $\begin{array}{l}\text { A Comparative Analysis } \\
\text { of Comprehensive } \\
\text { Geriatric Assessments } \\
\text { for Nursing Home } \\
\text { Residents Receiving } \\
\text { Palliative Care: A } \\
\text { Systematic Review }\end{array}$ & $\begin{array}{l}\text { Revisão de } \\
\text { literatura. }\end{array}$ & $\begin{array}{c}\text { Identificar todas as } \\
\text { avaliações geriátricas } \\
\text { usadas para cuidados } \\
\text { paliativos. }\end{array}$ & ILPI & Moderado \\
\hline $\begin{array}{l}\text { [08] Johnston, B. et al. / } \\
\text { 2018 / INGLATERRA / } \\
\text { BMC Palliative Care }\end{array}$ & $\begin{array}{c}\text { Impact ok the Macmillan } \\
\text { specialist Care at } \\
\text { Home service: } \text { a mixed }\end{array}$ & $\begin{array}{l}\text { Método } \\
\text { misto. }\end{array}$ & $\begin{array}{l}\text { Avaliar o processo e o } \\
\text { impacto na equipe, } \\
\text { pacientes e prestadores de }\end{array}$ & Domicílio & Moderado \\
\hline
\end{tabular}




\begin{tabular}{|c|c|c|c|c|c|}
\hline & $\begin{array}{l}\text { methods evaluation } \\
\text { across six sites. }\end{array}$ & & $\begin{array}{c}\text { cuidados de serviços de } \\
\text { Atendimento } \\
\text { Especializado. }\end{array}$ & & \\
\hline $\begin{array}{c}\text { [09] Stajduhar, K. et al. } \\
\text { / 2011 /CANADÁ / } \\
\text { Journal of Advanced } \\
\text { Nursing }\end{array}$ & $\begin{array}{c}\text { Home care nurses' } \\
\text { decisions about the need } \\
\text { for and amount } \\
\text { of 7iloto f at the 7iloto } \\
\text { life. }\end{array}$ & $\begin{array}{l}\text { Estudo } \\
\text { qualitativo. }\end{array}$ & $\begin{array}{l}\text { Explorar a tomada de } \\
\text { decisão sobre a } \\
\text { necessidade e a } \\
\text { quantidade de serviço por } \\
\text { clientes e famílias. }\end{array}$ & Domicílio & Moderado \\
\hline $\begin{array}{l}\text { [10] Faller, J. et al. / } \\
\text { 2016 / BRASIL / } \\
\text { Cogitare Enferm. }\end{array}$ & $\begin{array}{c}\text { Escala multidimensional } \\
\text { na avaliação da dor e } \\
\text { sintomas de } \\
\text { idosos em cuidados } \\
\text { paliativos. }\end{array}$ & $\begin{array}{l}\text { Estudo } \\
\text { quantitativo } \\
\text { de corte } \\
\text { transversal. }\end{array}$ & $\begin{array}{l}\text { Avaliar a dor e sintomas } \\
\text { associados em idosos com } \\
\text { câncer. }\end{array}$ & Domicílio & Baixo \\
\hline $\begin{array}{l}\text { [11] Duursma, F. et al. / } \\
2011 \text { / HOLANDA / } \\
\text { BMC Palliative Care }\end{array}$ & $\begin{array}{c}\text { Study protocol: } \\
\text { optimization of complex } \\
\text { palliative } \\
\text { care at home via } \\
\text { telemedicine. A cluster } \\
\text { randomized controlled } \\
\text { trial. }\end{array}$ & $\begin{array}{l}\text { Estudo } \\
\text { randomizado } \\
\text { controlado } \\
\text { por cluster. }\end{array}$ & $\begin{array}{l}\text { Investigar a eficácia da } \\
\text { teleconsulta em cuidados } \\
\text { paliativos domiciliares. }\end{array}$ & Domicílio & Baixo \\
\hline $\begin{array}{c}\text { [12] Little, S. et al. / } \\
2019 \text { / INGLATERRA / } \\
\text { British Journal of } \\
\text { Community Nursing }\end{array}$ & $\begin{array}{l}\text { Managing deterioration } \\
\text { in older adults in care } \\
\text { homes: a quality } \\
\text { improvement 7iloto to } \\
\text { introduce na 7ilot } \\
\text { warning tool }\end{array}$ & $\begin{array}{l}\text { Estudo } \\
\text { qualitativo } \\
\text { com o } \\
\text { método de } \\
\text { planejar, } \\
\text { fazer, } \\
\text { estudar, agir. }\end{array}$ & $\begin{array}{c}\text { Introdução de uma } \\
\text { ferramenta de alerta } \\
\text { precoce, para facilitar a } \\
\text { identificação e o } \\
\text { gerenciamento da } \\
\text { deterioração nos } \\
\text { residentes em casas de } \\
\text { repouso. }\end{array}$ & ILPI & Baixo \\
\hline $\begin{array}{c}\text { [13] Esteban-Burgos, A. } \\
\text { A. et al. /2019/ } \\
\text { ESPANHA / } \\
\text { Gerontology }\end{array}$ & $\begin{array}{c}\text { Prognostic Models } \\
\text { Associated with 6-Month } \\
\text { Survival of Patients } \\
\text { Admitted tô Nursing } \\
\text { Homes }\end{array}$ & $\begin{array}{c}\text { Estudo } \\
\text { prospectivo } \\
\text { longitudinal } \\
\text { observaciona } \\
\text { le analítico } \\
\text { de coorte. } \\
\end{array}$ & $\begin{array}{l}\text { Comparar a utilidade, em } \\
\text { lares de idosos, de dois } \\
\text { modelos de prognósticos } \\
\text { de sobrevida em seis } \\
\text { meses. }\end{array}$ & ILPI & Baixo \\
\hline $\begin{array}{c}\text { [14] Roberts, D. et al. / } \\
\text { 2014 / CANADÁ / } \\
\text { Home Healthc Nurse }\end{array}$ & $\begin{array}{c}\text { Applying Research into } \\
\text { practice: A guide to } \\
\text { determine the next } \\
\text { palliative home care } \\
\text { nurse visit. }\end{array}$ & $\begin{array}{c}\text { Estudo } \\
\text { qualitativo. }\end{array}$ & $\begin{array}{l}\text { Informar as avaliações } \\
\text { clínicas, julgamentos e } \\
\text { decisões para determinar } \\
\text { a próxima visita de } \\
\text { enfermagem. }\end{array}$ & Domicílio & Muito baixo \\
\hline $\begin{array}{c}\text { [15] Rotter, B; Grant, } \\
\text { M. / } 2018 \text { / EUA / } \\
\text { Journal of Hospice \& } \\
\text { Palliative Nursing }\end{array}$ & $\begin{array}{l}\text { Symptom Assessment } \\
\text { and Hospital Utilization } \\
\text { in a Home-Based } \\
\text { Palliative Care } \\
\text { Program. }\end{array}$ & $\begin{array}{l}\text { Revisão de } \\
\text { literatura. }\end{array}$ & $\begin{array}{l}\text { Rastrear a gravidade dos } \\
\text { sintomas dos idosos em } \\
\text { CP e coletar dados de } \\
\text { utilização de escala. }\end{array}$ & Domicílio & Muito baixo \\
\hline $\begin{array}{l}\text { [16] Henoch, B. et al. / } \\
\text { 2019 / SUÉCIA / } \\
\text { Upsala Journal of } \\
\text { Medical Sciences }\end{array}$ & $\begin{array}{l}\text { Equal palliative care for } \\
\text { patients with COPD? A } \\
\text { 7iloto f7s register study }\end{array}$ & $\begin{array}{l}\text { Estudo } \\
\text { retrospectivo } \\
\text { de registro } \\
\text { nacional. }\end{array}$ & $\begin{array}{c}\text { Comparar pacientes com } \\
\text { DPOC nos CP } \\
\text { Especializados com } \\
\text { aqueles nos asilos. }\end{array}$ & $\begin{array}{c}\text { ILPI e } \\
\text { domicílio }\end{array}$ & Muito Baixo \\
\hline $\begin{array}{l}\text { [17] Kirsten, H et al. / } \\
\text { 2014/ BÉLGICA/ } \\
\text { Palliative and } \\
\text { Supportive Care }\end{array}$ & $\begin{array}{c}\text { Does using the interRAI } \\
\text { Palliative Care } \\
\text { instrument reduce the } \\
\text { needs and symptoms of } \\
\text { nursing home 7iloto f7s } \\
\text { receiving palliative } \\
\text { care? }\end{array}$ & $\begin{array}{l}\text { Um estudo } \\
\text { pré e pós- } \\
\text { teste quase- } \\
\text { experimental } \\
\text {. }\end{array}$ & $\begin{array}{l}\text { Avaliar se os sintomas em } \\
\text { cuidados paliativos são } \\
\text { reduzidos após o uso de } \\
\text { ferramenta. }\end{array}$ & ILPI & Muito Baixo \\
\hline $\begin{array}{l}\text { [18] Mitchell, G. et al. / } \\
2016 \text { / AUSTRÁLIA / } \\
\text { BMC Palliative Care }\end{array}$ & $\begin{array}{c}\text { Evaluation of a } 7 \text { iloto } f \\
\text { nurse practitioner led, } \\
\text { GP supported rural } \\
\text { palliative care } \\
\text { provision. }\end{array}$ & $\begin{array}{l}\text { Estudo } \\
\text { coorte. }\end{array}$ & $\begin{array}{l}\text { Desenvolver e executar } \\
\text { um modelo adequado de } \\
\text { prestação de cuidados. }\end{array}$ & Domicilio & Muito baixo \\
\hline $\begin{array}{c}\text { [19] Wenger, N. et al. / } \\
2011 \text { / EUA / J Am } \\
\text { Geriatr Soc }\end{array}$ & $\begin{array}{c}\text { Quality of Care } \\
\text { Provided in a Special } \\
\text { Needs Plan Using a } \\
\text { Nurse } \\
\end{array}$ & $\begin{array}{l}\text { Estudo } \\
\text { qualitativo, } \\
\text { retrospectivo } \\
\text {. }\end{array}$ & $\begin{array}{c}\text { Avaliar a qualidade da } \\
\text { assistência prestada a } \\
\text { idosos complexos e o } \\
\text { modelo de gerenciamento }\end{array}$ & Domicílio & Muito baixo \\
\hline
\end{tabular}




\begin{tabular}{|l|c|c|c|l|l|}
\hline & Care Manager Model. & & $\begin{array}{c}\text { de cuidados de } \\
\text { enfermagem no ambiente } \\
\text { comunitário. }\end{array}$ & & \\
\hline
\end{tabular}

Legenda: Cuidados Paliativos (CP); Doença Pulmonar Obstrutiva Crônica (DPOC); Instituição de Longa Permanência para Idosos (ILPI); Promoting Effective Advance Care for Elders (PEACE); Estados Unidos da America (EUA); Resident Assessment Instrument for Home Care (RAI-HC.). Fonte: Autores, com os dados da pesquisa.

Entre os estudos analisados, onze (57,9\%) tiveram como principal cenário de realização do cuidado o domicílio convencional/familiar dos pacientes, cinco (26,3\%) foram realizados em ILPIs e três $(15,7 \%)$ incluíram tanto o domicílio quanto a ILPI. Em relação à origem da pesquisa três estudos foram realizados no Canadá (15,7\%), três nos Estados Unidos $(15,7 \%)$, dois na Austrália (10,5\%), dois na Inglaterra (10,5\%), dois na Bélgica (10,5\%), dois na Suécia (10,5\%), um no Brasil $(5,2 \%)$, um na Holanda (5,2\%), um na Finlândia (5,2\%), um na Espanha (5,2\%) e um na Irlanda do Norte (5,2\%). Quanto ao idioma, $18(94,7 \%)$ dos artigos estavam disponíveis para a leitura em inglês e um $(5,3 \%)(03)$ em português.

Quanto ao ano de publicação, três foram publicados em 2011 (15,7\%), um em 2012 (5,2\%), um em 2013 (5,2\%), três em 2014 (15,7\%), um em 2015 (5,2\%), dois em 2016 (10,5\%), dois em 2018 (10,5\%) e seis em 2019 (31,5\%), mostrando aumento do interesse pelo tema. Foram diversos aos métodos utilizados e o mais frequente foi o estudo randomizado com pequenas variações, seguido das revisões de literatura. Após a análise do nível de evidência, dois artigos (10,5\%) foram considerados como alto; sete artigos $(36,8 \%)$ como moderados; quatro (21\%), como baixo e seis artigos $(31,5 \%)$ como muito baixo.

Dos artigos analisados, extraiu-se 42 instrumentos, dos quais 11 para avaliação dos sintomas [03, 04, 05, 06,10, 11, $13,14,15,16,18]$ e oito específicos para avaliação de cuidados paliativos $[03,07,08,11,13,14,17,18]$. Em cinco artigos foi identificado uso de instrumentos de avaliação da qualidade de vida [03, 04, 05, 07, 16], e em três estudos utilizou-se instrumentos de avaliação dos cuidadores [08, 11, 18]. Os mais citados foram o Instrumento Edmonton Symptom Assessment System (ESAS) [03, 10, 11, 13, 15], a Escala Australiana Karnofsky Performance Status (AKPS) [04, 13, 18], a Escala Hospitalar de Ansiedade e Depressão (HADS) [05, 11, 18], a Escala de Resultados de Cuidados Paliativos (POS) [07, 17, 18] e a Escala de Desempenho Paliativo (PPS) [08, 13, 14].

Para orientar a discussão, elaborou-se um quadro com os instrumentos de avaliação agrupados em quatro categorias conforme o foco da atuação do enfermeiro (Quadro 3).

Quadro 3 - Instrumentos adotados, categorias de avaliação e uso da enfermagem aplicado a idosos em cuidado paliativo domiciliar, Juiz de Fora, Brasil, 2021.

\begin{tabular}{|c|c|c|}
\hline Instrumentos de Avaliação Utilizados & Categoria & $\begin{array}{c}\text { Uso no Processo de Cuidar da } \\
\text { Enfermagem }\end{array}$ \\
\hline $\begin{array}{l}\text { ESAS, [03, 10, 15]; CDR [03]; MMSE [03]; MNA [03]; } \\
\text { IMC [03]; PAINAD [03]; Instrumento 15D para avaliação } \\
\text { da QVRS [03]; SWC-OLD [03]; ICC [03]; RAI-HC [06]; } \\
\text { Escala CHESS incorporada ao RAI-HC [06]; HADS [18]; } \\
\text { POS [07, 18]; AKPS [18]; CSNAT [18]; FAMCARE } \\
\text { [18]; MVQOLI-R [07]; mQOLC-E [07]; MQLS [07]; } \\
\text { interRAI PC [07]. HADS [05]; Memorial Symptom } \\
\text { Escala de Avaliação [05]; QUAL-E [05]; } \\
\text { Relacionamentos (Meaning in Life) [05]; Medida de } \\
\text { Ativação do Paciente [05]; AVD [02]; AIVD [02]; MMSE } \\
\text { [02]; InterRAI PC [17]; POS [17]. }\end{array}$ & $\begin{array}{c}\text { Avaliação multidimensional } \\
\text { do idoso em cuidado paliativo } \\
\text { domiciliar }\end{array}$ & $\begin{array}{l}\text { Ações assistenciais baseadas em } \\
\text { Avaliação Geriátrica Ampla de sintomas } \\
\text { físicos, competência cognitiva, humor, } \\
\text { comunicação, status funcional, } \\
\text { espiritualidade, aplicando instrumentos de } \\
\text { avaliação; protocolos baseados em } \\
\text { evidências. } \\
{[02,03,05,06,07,10,15,17,18]}\end{array}$ \\
\hline
\end{tabular}




\begin{tabular}{|c|c|c|}
\hline $\begin{array}{l}\text { ACOVE [19]; RAI-HC [06]; Escala CHESS incorporada } \\
\text { ao RAI-HC [06]. }\end{array}$ & & $\begin{array}{l}\text { Ações assistenciais considerando: riscos } \\
\text { de quedas, incontinência urinária, status } \\
\text { de vacinação e triagem de causas } \\
\text { reversíveis de desnutrição, dor, cognição, } \\
\text { comunicação, saúde mental e } \\
\text { funcionalidade em idosos com demências. } \\
{[01,06,19]}\end{array}$ \\
\hline $\begin{array}{c}\text { PPS [14]; Escala de classificação da dor [14]; Ferramenta } \\
\text { de alerta precoce "significativo 7" [12]. }\end{array}$ & \multirow{6}{*}{$\begin{array}{l}\text { Avaliação para o cuidado } \\
\text { integral, acompanhamento do } \\
\text { luto e da satisfação do } \\
\text { cuidador e idoso em cuidado } \\
\text { paliativo domiciliar }\end{array}$} & $\begin{array}{l}\text { Ações realizadas conforme a } \\
\text { intensificação dos sintomas; humor, } \\
\text { confusão, dor, hidratação, pele, respiração } \\
\text { e hábitos de higiene. }[12,14]\end{array}$ \\
\hline $\begin{array}{c}\text { ESAS [11]; HADS [11]; PNPC [11]; NCQ [11]; EDIZ } \\
\text { [11]. Satisfação do paciente e do cuidador com a } \\
\text { teleconsulta [11]; IPP [08]; PPS [08]; IPOS [08]; CSNAT } \\
\text { [08]; POS [18]; AKPS [18]; CSNAT [18]; FAMCARE } \\
\text { [18]. }\end{array}$ & & $\begin{array}{l}\text { Cuidados clínicos com realização de } \\
\text { procedimentos domiciliares: manejo da } \\
\text { analgesia e efeitos colaterais; transfusões } \\
\text { de sangue, antibióticos IV ou } \\
\text { bifosfonatos, ultrassom, analgesia } \\
\text { intratecal; prevenção de quedas, lesões } \\
\text { por pressão, infecções; provisão de } \\
\text { auxílios à mobilidade ou oxigênio } \\
\text { suplementar; realiza a primeira consulta; } \\
\text { segue protocolo de consulta predefinido. } \\
{[01,08,11,18]}\end{array}$ \\
\hline $\begin{array}{c}\text { ESAS [03]; HADS [05]; PPS [14]; Escala de classificação } \\
\text { da dor [14]; AKPS [04]; Qualidade de Vida (McGill QOL } \\
\text { Questionnaire) [04]; Inventário Breve de Dor (escala } \\
\text { numérica) [04]; Memorial Symptom Escala de Avaliação } \\
\text { [04,05]; QUAL-E [05]; Relacionamentos (Meaning in } \\
\text { Life) [05]; Medida de Ativação do Paciente [05]; CDR } \\
\text { [03]; MMSE [03]; MNA [03]; IMC [03]; PAINAD [03]; } \\
\text { Instrumento 15D para avaliação da QVRS [03]; SWC- } \\
\text { OLD [03]; ICC [03]; mMRC [16]; QVRS e Clinical } \\
\text { COPD Questionnaire [16]; CAT [16]. }\end{array}$ & & $\begin{array}{l}\text { Cuidados e gerenciamento de } \\
\text { situações/preocupações; de mudanças que } \\
\text { estão ocorrendo; de necessidades e } \\
\text { enfrentamento frente à doença; suporte } \\
\text { emocional; e acompanhamento do luto } \\
\text { familiar; avalia capacidade e disposição } \\
\text { do cuidador para o cuidado através de } \\
\text { fatores como idade, conhecimentos, } \\
\text { estado emocional, confiança, } \\
\text { disponibilidade, experiência; estabelece } \\
\text { confiança com o paciente e cuidador. [03, } \\
04,05,09,14,16]\end{array}$ \\
\hline $\begin{array}{l}\text { ESAS [13]; mMRC [16]; QVRS e Clinical COPD } \\
\text { Questionnaire [16]; CAT [16]; IPP [08, 13]; PPS [08, 13]; } \\
\text { KPS [13]; ferramenta NECPAL [13]; PIG [13]; ICC [13]; } \\
\text { IPOS [08]; CSNAT [08]. }\end{array}$ & & $\begin{array}{l}\text { Ações conforme a necessidade de } \\
\text { cuidados paliativos físicos psicológicos, } \\
\text { emocionais, espirituais, de informação e } \\
\text { de apoio. }[08,13,16]\end{array}$ \\
\hline $\begin{array}{c}\text { ESAS [15]; mMRC [16]; QVRS e Clinical COPD } \\
\text { Questionnaire [16]; CAT [16]; RAI-HC [06]; Escala } \\
\text { CHESS incorporada ao RAI-HC [06]. }\end{array}$ & & $\begin{array}{l}\text { Ações de apoio psicossocial e existencial, } \\
\text { incluindo condições de isolamento social, } \\
\text { angústia do cuidador. }[06,15,16]\end{array}$ \\
\hline $\begin{array}{c}\text { AKPS [04]; Qualidade de Vida (McGill QOL } \\
\text { Questionnaire) [04]; Inventário Breve de Dor (escala } \\
\text { numérica) [04]; Memorial Symptom Escala de Avaliação } \\
\text { [04]. }\end{array}$ & & $\begin{array}{l}\text { Ações para alívio da dor e educativas com } \\
\text { paciente e cuidador visando a qualidade } \\
\text { de vida. [04] }\end{array}$ \\
\hline $\begin{array}{c}\text { ESAS [03, 13, 15]; HADS [05]; Memorial Symptom } \\
\text { Escala de Avaliação [05]; QUAL-E [05]; } \\
\text { Relacionamentos (Meaning in Life) [05]; Medida de } \\
\text { Ativação do Paciente [05]; ACOVE [19]; CDR [03]; } \\
\text { MMSE [03]; MNA [03]; IMC [03]; PAINAD [03]; } \\
\text { Instrumento 15D para avaliação da QVRS [03] SWC- } \\
\text { OLD [03]; ICC [03]; IPP [08]; PPS [08]; IPOS [08]; } \\
\text { CSNAT [08, 18]; POS [18]; AKPS [18]; FAMCARE } \\
\text { [18]. }\end{array}$ & \multirow{3}{*}{$\begin{array}{c}\text { Avaliação para gerenciamento } \\
\text { do cuidado paliativo ao idoso } \\
\text { no domicílio e na rede }\end{array}$} & $\begin{array}{l}\text { Ações de gerenciamento dos cuidados } \\
\text { paliativos e prestação de cuidados } \\
\text { práticos ao final da vida, como } \\
\text { administração de medicamentos; apoio } \\
\text { psicossocial e emocional. [03, } 04,05,08, \\
15,18,19]\end{array}$ \\
\hline $\begin{array}{l}\text { HADS [05]; Memorial Symptom Escala de Avaliação } \\
\text { [05]; QUAL-E [05]; Relacionamentos (Meaning in Life) } \\
\text { [05]; Medida de Ativação do Paciente [05]; mMRC [16]; } \\
\text { QVRS e Clinical COPD Questionnaire [16]; CAT [16]; } \\
\text { Ferramenta de alerta precoce "significativo 7" [12]; RAI- } \\
\text { HC [06]; Escala CHESS incorporada ao RAI-HC [06]; }\end{array}$ & & $\begin{array}{l}\text { Gerenciamento de sintomas, nutrição; } \\
\text { polifarmácia; cuidados domiciliares e } \\
\text { atendimento a necessidades físicas. [05, } \\
06,12,16]\end{array}$ \\
\hline $\begin{array}{c}\text { ESAS-r [11]; HADS [11]; PNPC [11]; NCQ [11]; EDIZ } \\
\text { [11]; Satisfação do paciente e do cuidador com a }\end{array}$ & & $\begin{array}{l}\text { Ações para integrar cuidados de longo } \\
\text { prazo com base na comunidade; atua }\end{array}$ \\
\hline
\end{tabular}




\begin{tabular}{|c|c|c|}
\hline $\begin{array}{c}\text { teleconsulta [11]; Ferramenta de alerta precoce } \\
\text { "significativo 7" [12]; IPP [08]; PPS [08]; IPOS [08]; } \\
\text { CSNAT [08]. }\end{array}$ & & $\begin{array}{l}\text { prevenindo hospitalizações } \\
\text { desnecessárias; gerencia problemas nos } \\
\text { cuidados paliativos, desenvolve } \\
\text { protocolos de cuidado. }[08,11,12]\end{array}$ \\
\hline $\begin{array}{l}\text { ESAS [03]; CDR [03]; MMSE [03]; MNA [03]; IMC } \\
\text { [03]; PAINAD [03]; Instrumento 15D para avaliação da } \\
\text { QVRS [03]; SWC-OLD [03]; ICC [03]; mMRC [16]; } \\
\text { QVRS e Clinical COPD Questionnaire [16]; CAT [16]. }\end{array}$ & \multirow{3}{*}{$\begin{array}{l}\text { Avaliação para gerenciamento } \\
\text { e elaboração de plano de } \\
\text { cuidados em equipe } \\
\text { multiprofissional }\end{array}$} & $\begin{array}{l}\text { Utiliza comunicação eficaz com a equipe } \\
\text { paciente e família }[01,03,16]\end{array}$ \\
\hline $\begin{array}{c}\text { ESAS [03, 13]; PPS [14]; Escala de classificação da dor } \\
\text { [14]; HADS [05, 18]; Memorial Symptom escala de } \\
\text { avaliação [05]; QUAL-E [05]; Relacionamentos (Meaning } \\
\text { in Life) [05]; Medida de Ativação do Paciente [05]; } \\
\text { ACOVE [19]; CDR [03]; MMSE [03]; MNA [03]; IMC } \\
\text { [03]; PAINAD [03]; Instrumento 15D para avaliação } \\
\text { QVRS [03]; SWC-OLD [03]; ICC [03, 13]; IPP [13]; PPS } \\
\text { [08, 13]; KPS [13]; ferramenta NECPAL [13]; PIG [13]; } \\
\text { InterRAI PC [17]; POS [17, 18]; AKPS [18]; CSNAT } \\
\text { [18]; FAMCARE [18]; }\end{array}$ & & $\begin{array}{l}\text { Elaboração, gerenciamento e execução de } \\
\text { planejamento avançado de cuidados; com } \\
\text { abordagens de medicina geriátrica; } \\
\text { individualizado e com a equipe } \\
\text { multiprofissional. [03, 05,13,14,17,18, } \\
\text { 19] }\end{array}$ \\
\hline $\begin{array}{c}\text { ESAS [11]; PPS [14]; Escala de classificação da dor [14]; } \\
\text { AKPS [04]; Qualidade de Vida (McGill QOL } \\
\text { Questionnaire) [04]; Inventário Breve de Dor (escala } \\
\text { numérica) [04]; Memorial Symptom Escala de Avaliação } \\
\text { [04]; HADS [11]; PNPC [11]; NCQ [11]; EDIZ [11]. } \\
\text { Satisfação do paciente e do cuidador com a teleconsulta } \\
\text { [11]; AVD [02]; AIVD [02]; MMSE [02]. }\end{array}$ & & $\begin{array}{l}\text { Participa da discussão de casos; } \\
\text { planejamento e intervenção } \\
\text { interdisciplinar; aconselhamento o clínico } \\
\text { geral sobre a política de tratamento para o } \\
\text { paciente; atuação em equipe geriátrica } \\
\text { móvel que realiza visitas e cuidados na a } \\
\text { casa do paciente. }[01,02,04,11,14]\end{array}$ \\
\hline
\end{tabular}

Legenda: Edmonton Symptom Assessment System (ESAS); Escala Clinical Dementia Rating (CDR); Mini-Menta State Examination (MMSE); Avaliação Mininutricional (MNA); Î́ndice de Massa corporal (IMC); Avaliação da Dor na Demência Avançada (PAINAD); Qualidade de Vida Relacionada à Saúde (QVRS); Escala de fim de vida em demência (SWC-OLD); Índice de comorbidade de Charlson (ICC) Instrumento de Avaliação de Residente para Assistência Domiciliar (RAI-HC); Alterações na Saúde, Doença em Estágio Final, Sinais e Sintomas (Escala CHESS); Escala Hospitalar de Ansiedade e Depressão (HADS); Escala de Resultados Paliativos (POS); Australian Karnofsky Performance Scale (AKPS); Escala de Karnofsky (KPS); Ferramenta de avaliação de necessidades de apoio ao cuidador (CSNAT); Satisfação em Família com Cuidados Avançados em Câncer (FAMCARE); Índice de Qualidade de Vida Missoula- VITAS (MVQOLI-R); Questões de qualidade de vida modificadas no final da vida / Questionário (mQOLC-E); Escala de Qualidade de Vida McMaster (MQLS); Instrumento de cuidados Paliativos InterRAI (interRAI PC); Qualidade de vida / morte (QUAL-E); Atividades da Vida Diária (AVD); Atividades Instrumentais (AIVD); Instrumento Avaliação de Cuidados a Pessoas Idosas Vulneráveis (ACOVE); Escala de desempenho Paliativo (PPS); Escala de Dispneia do Conselho de Pesquisa Médica [modificada] (mMRC); Teste de Avaliação da DPOC (CAT); Índice Prognóstico Paliativo (IPP); Necessidades Paliativas (ferramenta NECPAL); Guia de Indicador Prognóstico (PIG); Escalas paliativas integradas de resultados (IPOS); Problemas e Necessidades em Cuidados Paliativos (PNPC); Continuidade da experiência do atendimento médico na última fase da vida: Nijmegen Continuity Questionnaire (NCQ); Sobrecarga do cuidador familiar: autopercepção da pressão do cuidado informal (EDIZ). Fonte: Autores, com os dados da pesquisa.

Um destaque desta revisão refere-se à utilização da avaliação multidimensional do idoso no cuidado paliativo domiciliar, fundamentando-se as ações assistenciais em avaliação geriátrica ampla, a qual inclui, além da avaliação clínica de sintomas, status de vacinação e triagem de causas reversíveis de desnutrição e dor, as avaliações relativas às síndromes geriátricas, envolvendo status funcional, riscos de quedas; incontinência urinária; competência cognitiva e funcionalidade em idosos com demências; humor e aspectos da saúde mental; comunicação e espiritualidade, aplicando instrumentos próprios e adotando protocolos baseados em evidências (Allen et al., 2012; Burns \& McIlfatrick, 2015; Faller, Zilly, Moura, \& Brusnicki, 2016; Fristedt, Nystedt, \& Skogar, 2019; Harman et al., 2019; Hermans et al., 2014, 2018; Lamppu et al., 2019; Mitchell et al., 2016; Rotter \& Grant, 2018; Stajduhar et al., 2011; Wenger et al., 2011).

A avaliação multidimensional do idoso em $\mathrm{CP}$ conta com instrumentos específicos, que consideram e procuram pormenorizar as peculiaridades do envelhecimento humano, para cada área do conhecimento como as condições nutricionais, funcionais, presença de dor. Por meio do gerenciamento clínico feito pelo médico e coordenação dos cuidados feita pelo enfermeiro, e com a participação por área de atuação multiprofissional geriátrica para a realização de avaliações detalhadas, o modelo proposto pelo autor torna possível criar um plano de cuidados integral, o que promove qualidade de vida ao idoso, família e cuidadores (Veras, 2020).

É preciso considerar que o processo de envelhecimento humano acarreta inúmeras mudanças fisiológicas, psicológicas e sociais para o indivíduo, o que, somado a condições patológicas, pode resultar em demandas de CP. O cuidado domiciliar possibilita que o enfermeiro preste um atendimento de qualidade, com acompanhamento do desempenho paliativo, 
percepção de sinais e manejo correto dos sintomas, possibilitando-que o idoso desfrute de um processo de finitude digno e respeitoso (Marques \& Bulgarelli, 2020).

A oferta dos cuidados paliativos multiprofissionais no ambiente domiciliar permite ao idoso experienciar a sua finitude de vida em seu convívio familiar, realizando uma avaliação multidimensional de saúde da pessoa idosa, ao mesmo tempo em que recebe assistência especializada. Estudos tem recomendado ser essencial que a equipe de atendimento esteja qualificada para o acolhimento, cuidados e orientações tanto ao paciente quanto à sua família e cuidadores (Meireles et al., 2020). Uma das maneiras de se realizar a avaliação multidimensional de saúde da pessoa idosa no domicílio em CP é através dos registros clínicos, quando é possível conhecer a qualidade dos cuidados que foram oferecidos em final de vida, e identificar as áreas que necessitam de melhorias (Mestre \& Silva, 2020).

Todavia, predomina a avaliação segundo os princípios dos cuidados paliativos que orienta a assistência pautada em cuidados clínicos com realização de procedimentos no domicílio, dentre os quais se destacam o manejo da analgesia e efeitos colaterais; transfusões de sangue, antibioticoterapia endovenosa, analgesia intratecal; prevenções de quedas, lesões por pressão e infecções; provisão de auxílios à mobilidade ou ao uso de oxigênio suplementar; seguindo protocolo de consulta predefinido. Além dos cuidados clínicos a avaliação fundamenta ações conforme as situações, preocupações e mudanças que estão ocorrendo; necessidades educativas e de informação, de apoio psicossocial, espiritual, existencial, social e de enfrentamento da doença. Ressalta-se preocupação com a qualidade de vida e atenção baseada no estabelecimento de confiança com paciente e cuidador; reconhecimento da angústia, capacidade e disposição do cuidador para o cuidado considerando-se a idade, conhecimentos, estado emocional, confiança, disponibilidade e experiência. Além disso, inclui o acompanhamento do luto familiar após o óbito do idoso (Abernethy et al., 2013; Allen et al., 2012; Burns \& Mcllfatrick, 2015; Duursma, Schers, Vissers, \& Hasselaar, 2011; Esteban-Burgos et al., 2019; Harman et al., 2019; Henoch, Strang, Löfdahl, \& Ekberg-Jansson, 2019; Johnston et al., 2018; Lamppu et al., 2019; Mitchell et al., 2016; Roberts, McLeod, Stajduhar, Webber, \& Milne, 2014; Rotter \& Grant, 2018; Stajduhar et al., 2011).

Aspectos que podem conferir a qualidade da avaliação em cuidados paliativos de enfermagem tem sido relacionado a oferta de cuidado holístico, com destaque para as relações interpessoais, comunicação e ao apoio espiritual. Para os cuidadores foi mais importante o tempo de dedicação dos profissionais e o acesso a eles. A avaliação de enfermagem sobretudo sob o auxílio de escalas específicas e validadas, foi considerado como um parâmetro para a boa enfermagem no campo dos cuidados paliativos. Esse foi um marco importante para a profissão ao envolver o reconhecimento da autonomia profissional no cuidado e na busca constante por estabelecer indicadores capazes de orientar a prática. $O$ estudo concluiu que os enfermeiros precisam utilizar instrumentos que facilitem o conhecimento das atividades rotineiras voltadas ao cuidado aos idosos em final de vida e aos seus familiares (Zulueta Egea, Prieto-Ursúa, \& Bermejo Toro, 2020).

O gerenciamento dos problemas nos cuidados paliativos e a prestação de cuidados práticos ao final da vida, também nortearam a avaliação da enfermagem, considerando-se as ações para integrar cuidados de longo prazo com base na comunidade, a prevenção de hospitalizações desnecessárias e o desenvolvimento de protocolos de cuidado (Abernethy et al., 2013; Allen et al., 2012; Harman et al., 2019; Johnston et al., 2018; Lamppu et al., 2019; Little, Rodgers, \& Fitzpatrick, 2019; Mitchell et al., 2016; Rotter \& Grant, 2018; Wenger et al., 2011).

Os enfermeiros, por desempenharem papéis de relevância na gestão do sofrimento de idosos em cuidados paliativos, ao promover a prevenção e alívio dos sintomas, necessitam de instrumentos validados para o gerenciamento dos sintomas, que são uteis para facilitar a triagem, monitorar o progresso e orientar as decisões de tratamento de final de vida (GutiérrezSánchez, Gómez-García, Cuesta-Vargas, \& Pérez-Cruzado, 2020).

Em um grupo de artigos a avaliação da enfermagem considerou o gerenciamento e elaboração de plano de cuidados multiprofissional tendo por base a comunicação eficaz com a equipe paciente e família e o gerenciamento e execução de 
planejamento avançado de cuidados. Busca-se apoiar nas abordagens geriátricas para a atuação individualizada e com a equipe. A avaliação direciona a atuação da equipe geriátrica móvel que realiza visitas e cuidados na casa do paciente; as discussões de casos; o planejamento e intervenções interdisciplinares e o aconselhamento ao clínico geral sobre a política de tratamento (Abernethy et al., 2013; Allen et al., 2012; Burns \& McIlfatrick, 2015; Duursma et al., 2011; Esteban-Burgos et al., 2019; Fristedt et al., 2019; Henoch et al., 2019; Hermans et al., 2018; Lamppu et al., 2019; Mitchell et al., 2016; Roberts et al., 2014; Wenger et al., 2011).

A aproximação entre profissionais de saúde e famílias foi identificada como eixo condutor do cuidado, e mostrou- se fundamental para a criação de um elo concreto de comunicação entre a família, a rede de cuidado que envolve o idoso em sua finitude, e o serviço de saúde. Foi identificado a necessidade de troca interna em comunicação entre a equipe multiprofissional para a promoção de melhorias do cuidado. A proximidade da equipe com a família criou um vínculo humano de empatia, também, por parte do profissional que buscou conhecer a realidade de quem necessita de cuidados paliativos, em um contexto de qualidade de morte. O profissional de saúde se identifica com o sofrimento da família e realiza práticas para amenizá-lo, por meio da transmissão de segurança e conforto (Marques \& Bulgarelli, 2020).

A comunicação tem um papel essencial nas práticas, por colaborar na formação dos vínculos, para que o cuidador assuma o seu papel ao permitir o final de vida na fase terminal a acontecer em casa, junto a família. A comunicação efetiva foi reconhecida como fator que influenciou a percepção das equipes de que os pacientes não estavam preparados para a discussão sobre o fim de suas vidas. O medo enfrentado pelo profissional de saúde quanto a possibilidade de tirar as esperanças de um doente, e a falta de formação especializada foram os desafios destacados envolvendo a comunicação. O incentivo a melhores práticas de comunicação entre profissionais de saúde com os pacientes, assim como a incorporação deles para a tomada de decisão são fatores que influenciam a sobrevida dos doentes terminais em domicílio (Mestre \& Silva, 2020).

Além das categorias analisadas destaca-se a avaliação de idosos em cuidados paliativos que residem em ILPI, pautando-se em avaliação geriátrica ampla (instrumento interRAI PC (07)), orientando o planejamento avançado do cuidado, baseado em comunicação e suporte emocional à família e ao idoso (escalas de cuidados paliativos IPP, PPS, KPS (13, 08); na prevenção de hospitalizações e na identificação precoce de sinais e sintomas; prevenção de quedas, lesões por pressão e infecções (Ferramenta de alerta precoce "significativo 7" (12).

\section{Conclusão}

Esta revisão mostrou que a avaliação de enfermagem se imbuiu de princípios holísticos para a identificação de necessidades de cuidados paliativos de idosos no domicílio, com destaque para a avaliação clínica, sobressaindo-se a avaliação da dor, aspectos psicossociais, e espirituais. A avaliação multidimensional do idoso ocorreu associada às avaliações próprias dos cuidados paliativos e a comunicação eficaz da enfermagem com a equipe interdisciplinar, idosos e familiares foi mencionada como um fator importante para a avaliação e desenvolvimento dos cuidados paliativos. Houve também uso de instrumentos de avaliação visando o gerenciamento dos cuidados paliativos pela enfermagem e em equipe multiprofissional.

Conclui-se que na avaliação de idosos em cuidados paliativos domiciliares sobressai a avaliação clínica focada nos sintomas e na sua intensidade, na vacinação, na prevenção da desnutrição apoiando a realização de procedimentos complexos, acompanhamento do idoso com práticas de enfermagem, provisão de $\mathrm{O} 2$ e auxílio à mobilidade. Entremeada a estas, avaliamse aspectos psicossociais e espirituais, buscando-se a elaboração de plano de cuidados de enfermagem ou multiprofissional centrado no apoio ao enfrentamento do idoso e família, na angústia e capacidade de cuidar do cuidador, em intervenção interdisciplinar e junto à equipe. Destaca, todavia, a inclusão da avaliação multidimensional do idoso, associada a abordagem gerontológica ao idoso em cuidado paliativo domiciliar, com o gerenciamento de polifarmácia; promoção da autonomia do 
idoso, promoção de confiança e atenção ao cuidador. Ressaltam-se a valorização dos cuidados com base na comunidade e o uso de protocolo baseado em evidências.

O presente estudo permitiu compreender, de forma ampla, um dos eixos de atuação da enfermagem no contexto dos cuidados paliativos domiciliares, a partir de experiências nacionais e internacionais. Ficou evidenciada a articulação de diversas ações como a avaliação multidimensional por meio do uso de instrumentos e, a prestação de cuidados diretos e indiretos ao idoso, incluindo a realização de procedimentos. Ao considerar a relevância da temática sugere-se que sejam desenvolvidos novos estudos relacionados ao processo de trabalho e atuação da enfermagem no cenário domiciliar, no âmbito dos cuidados paliativos, vislumbrando atualização contínua para o cuidado do enfermeiro, além de fortalecer seu papel na construção do conhecimento científico.

\section{Referências}

Abernethy, A. P., Currow, D. C., Shelby-James, T., Rowett, D., May, F., Samsa, G. P., \& Phillips, P. A. (2013). Delivery Strategies to Optimize Resource Utilization and Performance Status for Patients With Advanced Life-Limiting Illness: Results From the "Palliative Care Trial" [ISRCTN 81117481]. Journal of Pain and Symptom Management, 45(3), 488-505. https://doi.org/10.1016/j.jpainsymman.2012.02.024

Adamy, E. K., Zocche, D. A. de A., \& Almeida, M. de A. (2020). Revista Gaúcha de Enfermagem Contribuição do processo de enfermagem para construção identitária dos profissionais de enfermagem. Rev Gaúcha Enferm, 41, 20190143. https://doi.org/10.1590/1983-1447.2020.20190143

Allen, K. R., Hazelett, S. E., Radwany, S., Ertle, D., Fosnight, S. M., \& Moore, P. S. (2012). The Promoting Effective Advance Care for Elders (PEACE) Randomized Pilot Study: Theoretical Framework and Study Design. Population Health Management, 15(2), 71-77. https://doi.org/10.1089/pop.2011.0004

Barbosa, A. N., Nascimento, I. A. do, Carvalho, M. J. S. de, Aoyama, E. de A., \& Lima, R. N. de. (2019). A importância da assistência humanizada prestada pelo enfermeiro nos cuidados paliativos ao paciente oncológico terminal. Revista Brasileira Interdisciplinar de Saúde, 1(4), 92-96.

Brasil. Portaria No 2.528 de 19 de outubro de 2006 (2006).

Brasil. Portaria No 2.029 de 24 de agosto de 2011 (2011).

Brasil. (2014). Diretrizes metodológicas: elaboração de revisão sistemática e metanáçise de estudos observacionais comparativos sobre fatores de risco e prognóstico (1st ed.). Retrieved from www.saude.gov.br

Burns, M., \& McIlfatrick, S. (2015). Palliative care in dementia: literature review of nurses' knowledge and attitudes towards pain assessment. International Journal of Palliative Nursing, 21(8), 400-407. https://doi.org/10.12968/ijpn.2015.21.8.400

Caveião, C., Hey, A. P., Bernardes, K., Lara, R., Alfaro, A. S., \& Prestes, F. da S. (2019). Ações do enfermeiro em cuidados paliativos na oncologia : uma revisão integrativa Actions of the nurse in palliative care in oncology : an integrating review. Revista Saúde e Desenvolvimento, $13(16), 14$.

Donato, H., \& Donato, M. (2019). Stages for undertaking a systematic review. Acta Medica Portuguesa, 32(3), 227-235. https://doi.org/10.20344/amp.11923

Duursma, F., Schers, H. J., Vissers, K. C., \& Hasselaar, J. (2011). Study protocol: optimization of complex palliative care at home via telemedicine. A cluster randomized controlled trial. BMC Palliative Care, 10(1), 13. https://doi.org/10.1186/1472-684X-10-13

Enfermagem, conselho federal de. (2009). RESOLUÇÃO COFEN 358/2009.

Esteban-Burgos, A. A., El Mansouri-Yachou, J., Muñoz-Ramirez, R., Hueso-Montoro, C., Garcia-Caro, M. P., \& Montoya-Juarez, R. (2019). Prognostic Models Associated with 6-Month Survival of Patients Admitted to Nursing Homes. Gerontology, 65(1), 40-44. https://doi.org/10.1159/000490243

Faller, J. W., Zilly, A., Moura, C. B. de, \& Brusnicki, P. H. (2016). Escala multidimensional na avaliação da dor e sintomas de idosos em cuidados paliativos. Cogitare Enferm, 21(2), 1-10.

Fristedt, S., Nystedt, P., \& Skogar, Ö. (2019). Mobile Geriatric Teams - A Cost-Effective Way Of Improving Patient Safety And Reducing Traditional Healthcare Utilization Among The Frail Elderly? A Randomized Controlled Trial. Clinical Interventions in Aging, Volume 14, 1911-1924. https://doi.org/10.2147/CIA.S208388

Gardona, R. G. B., \& Barbosa, D. A. (2018). The importance of clinical practice supported by health assessment tools. Revista Brasileira de Enfermagem, 71(4), 1815-1816. https://doi.org/10.1590/0034-7167-2018710401

Gutiérrez-Sánchez, D., Gómez-García, R., Cuesta-Vargas, A. I., \& Pérez-Cruzado, D. (2020). The suffering measurement instruments in palliative care: A systematic review of psychometric properties. International Journal of Nursing Studies, 110. https://doi.org/10.1016/j.ijnurstu.2020.103704 
Harman, L. E., Guthrie, D. M., Cohen, J., Declercq, A., Fisher, K., Goodridge, D., \& Seow, H. (2019). Potential quality indicators for seriously ill home care clients: a cross-sectional analysis using Resident Assessment Instrument for Home Care (RAI-HC) data for Ontario. BMC Palliative Care, 18(1), 3. https://doi.org/10.1186/s12904-018-0389-y

Henoch, I., Strang, P., Löfdahl, C.-G., \& Ekberg-Jansson, A. (2019). Equal palliative care for patients with COPD? A nationwide register study. Upsala Journal of Medical Sciences, 124(2), 140-147. https://doi.org/10.1080/03009734.2019.1586803

Hermans, K., De Almeida Mello, J., Spruytte, N., Cohen, J., Van Audenhove, C., \& Declercq, A. (2014). A Comparative Analysis of Comprehensive Geriatric Assessments for Nursing Home Residents Receiving Palliative Care: A Systematic Review. Journal of the American Medical Directors Association, 15(7), 467-476. https://doi.org/10.1016/j.jamda.2014.01.002

Hermans, K., De Almeida Mello, J., Spruytte, N., Cohen, J., Van Audenhove, C., \& Declercq, A. (2018). Does using the interRAI Palliative Care instrument reduce the needs and symptoms of nursing home residents receiving palliative care? Palliative and Supportive Care, 16(1), 32-40. https://doi.org/10.1017/S1478951517000153

Johnston, B., Patterson, A., Bird, L., Wilson, E., Almack, K., Mathews, G., \& Seymour, J. (2018). Impact of the Macmillan specialist Care at Home service: a mixed methods evaluation across six sites. BMC Palliative Care, 17(1), 36. https://doi.org/10.1186/s12904-018-0281-9

Lamppu, P. J., Laurila, J., Finne-Soveri, H., Laakkonen, M.-L., Kautiainen, H., \& Pitkälä, K. H. (2019). Training nursing home staff to improve residents' endof-life care: design and baseline findings from a randomized controlled trial. European Geriatric Medicine, 10(4), 649-657. https://doi.org/10.1007/s41999019-00200-5

Larissa, K., Coutinho, B., \& Teixeira, F. B. (2021). Atenção domiciliar: Desafios para a promoção da saúde de idosos Home care: Challenges for the promotion of elderly health Atención en el hogar: Desafios para la promoción de la salud de los ancianos. 2021, 1-6.

Leandro, T. A., Alves, A. M., Pinheiro, A. K. B., Araujo, T. L. de, Quirino, G. da S., \& Oliveira, D. R. de. (2019). Nurses' competencies in health promotion for homebound older people. Revista Brasileira de Enfermagem, 72(suppl 2), 311-318. https://doi.org/10.1590/0034-7167-2018-0446

Little, S., Rodgers, G., \& Fitzpatrick, J. M. (2019). Managing deterioration in older adults in care homes: a quality improvement project to introduce an early warning tool. British Journal of Community Nursing, 24(2), 58-66. https://doi.org/10.12968/bjcn.2019.24.2.58

Malta, D. C., Bernal, R. T. I., Lima, M. G., Araújo, S. S. C. de, Silva, M. M. A. da, Freitas, M. I. de F., \& Barros, M. B. de A. (2017). Noncommunicable diseases and the use of health services: analysis of the National Health Survey in Brazil. Revista de Saúde Pública, 51(suppl 1). https://doi.org/10.1590/s15188787.2017051000090

Marques, F. P., \& Bulgarelli, A. F. (2020). The significance of home care in caring for the elderly in their twilight years: The human perspective of the SUS professional. Ciencia e Saude Coletiva, 25(6), 2063-2072. https://doi.org/10.1590/1413-81232020256.21782018

Meireles, D. S. de, Bittencourt, G. K. G. D., Montenegro, C. P. D., Fortunato, C. N., Melo, G. M. O. G. de, Soares, H. de S., \& Santos, M. S. dos. (2020). Assistência de enfermagem ao idoso em cuidados paliativos: um relato de experiência. Brazilian Journal of Development, 6(6), 40854-40867. https://doi.org/10.34117/BJDV6N6-576

Mestre, T. F. S., \& Silva, C. A. da. (2020). Realidades do mundo do trabalho de uma equipa comunitária de suporte em cuidados paliativos e as suas implicações nos cuidados aos doentes em trajetórias de fim de vida. Sociologia: Revista Da Faculdade de Letras Da Universidade Do Porto, 40, 35-56. https://doi.org/10.21747/08723419/soc40a2

Mitchell, G. K., Senior, H. E., Bibo, M. P., Makoni, B., Young, S. N., Rosenberg, J. P., \& Yates, P. (2016). Evaluation of a pilot of nurse practitioner led, GP supported rural palliative care provision. BMC Palliative Care, 15(1), 93. https://doi.org/10.1186/s12904-016-0163-y

Nunes De Moraes, E. (2012). ATENÇÃO À SAÚDE DO IDOSO: Aspectos Conceituais.

Okoli, C., Duarte, T. por:David W. A., \& Mattar, R. técnica e introdução:João. (2019). Guia Para Realizar uma Revisão Sistemática de Literatura. EaD Em Foco, 9(1). https://doi.org/10.18264/EADF.V9I1.748

Passarelles, D. M. do A., Rios, A. A., \& Santana, R. F. (2019). Diagnósticos de enfermería en cuidados paliativos oncológicos: revisión integrativa. Enfermeria Global, 18(3), 579-589. https://doi.org/10.6018/EGLOBAL.18.3.345201

Perreira, B. dos R., Jesus, I. M. O. de, \& Martins, M. M. F. (2020). Perfil sociodemográfico da mortalidade da população idosa no nordeste brasileiro. 18, 921. https://doi.org/https//doi.org/10.13037/ras.vol18n64.6273

Roberts, D., McLeod, B., Stajduhar, K. I., Webber, T., \& Milne, K. (2014). Applying Research Into Practice. Home Healthcare Nurse, 32(2), 88-95. https://doi.org/10.1097/NHH.0000000000000018

Rotter, B., \& Grant, M. (2018). Symptom Assessment and Hospital Utilization in a Home-Based Palliative Care Program. Journal of Hospice \& Palliative Nursing, 20(4), 332-337. https://doi.org/10.1097/NJH.0000000000000448

Sanvezzo, V. M. de S., Montandon, D. S., \& Esteves, L. S. F. (2018). Instrumentos de avaliação de funcionalidade de idosos em cuidados paliativos: uma revisão integrativa. Revista Brasileira Geriatria Gerontológica, 21(5), 627-638. https://www.scielo.br/pdf/rbgg/v21n5/pt_1809-9823-rbgg-21-05-00604.pdf

Stajduhar, K. I., Funk, L., Roberts, D., McLeod, B., Cloutier-Fisher, D., Wilkinson, C., \& Purkis, M. E. (2011). Home care nurses' decisions about the need for and amount of service at the end of life. Journal of Advanced Nursing, 67(2), 276-286. https://doi.org/10.1111/j.1365-2648.2010.05491.x 
Research, Society and Development, v. 10, n. 10, e520101018989, 2021

(CC BY 4.0) | ISSN 2525-3409 | DOI: http://dx.doi.org/10.33448/rsd-v10i10.18989

Torres, K. R. B. de O., Campos, M. R., Luiza, V. L., \& Caldas, C. P. (2020). Evolução das políticas públicas para a saúde do idoso no contexto do Sistema Único de Saúde. Physis: Revista de Saúde Coletiva, 30(1), 1-22. https://doi.org/10.1590/s0103-73312020300113

Veras, R. (2020). A contemporary and innovative care model for older adults. Revista Brasileira de Geriatria e Gerontologia, 23(1). https://doi.org/10.1590/1981-22562020023.200061

Wenger, N. S., Roth, C. P., Martin, D., Nickels, L., Beckman, R., Kamberg, C., \& Ganz, D. A. (2011). Quality of Care Provided in a Special Needs Plan Using a Nurse Care Manager Model. Journal of the American Geriatrics Society, 59(10), 1810-1822. https://doi.org/10.1111/j.1532-5415.2011.03599.x

Whittemore, R., \& Knafl, K. (2005). The integrative review: updated methodology. Journal of advanced nursing, 52(5), 546-553.

World Health Organization. (2018). Integrating palliative care and sympton relief into primary health care.

World Health Organization. (2020). Global Atlas Of Palliative Care. Retrieved from www.thewhpca.orgwww.thewhpca.org

Zulueta Egea, M., Prieto-Ursúa, M., \& Bermejo Toro, L. (2020). Good palliative nursing care: Design and validation of the palliative nursing care quality scale (PNCQS). Journal of Advanced Nursing, 76(10), 2757-2767. https://doi.org/10.1111/jan.14464 